\title{
Massive higher spin states in string theory and gravitational quadrupoles
}

\author{
Ioannis Giannakis* \\ Physics Department, The Rockefeller University, 1230 York Avenue, New York, New York 10021-6399 \\ and Department of Physics, New York University, 4 Washington Place, New York, New York 10003 ${ }^{\dagger}$ \\ James T. Liu ${ }^{\ddagger}$ \\ Physics Department, The Rockefeller University, 1230 York Avenue, New York, New York 10021-6399 \\ Massimo Porrati ${ }^{\S}$ \\ Physics Department, The Rockefeller University, 1230 York Avenue, New York, New York 10021-6399; \\ Department of Physics, New York University, 4 Washington Place, New York, New York 10003;" \\ and Theory Division, CERN CH 1211 Geneva 23, Switzerland
}

(Received 12 October 1998; published 19 April 1999)

\begin{abstract}
In this paper we study three point functions of the type II superstring involving one graviton and two massive states, focusing in particular on the spin- $\frac{7}{2}$ fermions at the first mass level. Defining a gravitational quadrupole " $h$ factor,', we find that the nonminimal interactions of string states in general are parametrized by $h \neq 1$, in contrast with the preferred field theory value of $h=1$ (for tree-level unitarity). This difference arises from the fact that consistent gravitational interactions of strings are related to the presence of a complete tower of massive states, not present in the ordinary field theory case. [S0556-2821(99)01908-6]
\end{abstract}

PACS number(s): 04.62.+v, 04.50.+h, 11.25.-w

\section{INTRODUCTION}

While the matter of consistent interactions of massive higher spin fields with gravity has been fairly well studied in the context of field theory [1], less is known in the case of string theory. Although for the latter, one may argue that strings must a priori present a consistent theory of gravity, it is nevertheless instructive to examine the nature of such interactions and to determine in particular how strings achieve such a consistency.

The simplest recipe for coupling massive fields to gravity (the "minimal coupling") is inherently ambiguous. Indeed, by replacing ordinary derivatives with covariant derivatives one is still free to add to the action terms that vanish on flat space; the commutator of two covariant derivatives, for instance. In Ref. [2] a prescription was given for fixing some of the ambiguities. There, by imposing tree-level unitarity up to the Planck scale on forward "Compton" scattering amplitudes of a single massive high-spin field, many coefficients in its action were fixed unambiguously. In particular, it was shown that starting from spin- $\frac{5}{2}$, tree-level unitarity requires the presence of terms proportional to the Riemann tensor in the three-point vertex describing single-graviton emission and absorption by the fermions.

Tree-level unitarity is really the statement that an interacting theory is weakly coupled up to a certain energy scale, $M_{\text {Planck }}$ or $\left(\alpha^{\prime}\right)^{-1 / 2}$, for instance. The recipe of Ref. [2] is not immediately applicable to string theory. The spectrum of

\footnotetext{
*Email: giannak@ theory.rockefeller.edu

${ }^{\dagger}$ Permanent address.

†Email address: jtliu@theory.rockefeller.edu

${ }^{\S}$ Email address: massimo.porrati@nyu.edu

"Permanent address.
}

string theory, indeed, contains many particles of everincreasing spin, some of which degenerate in mass. Thus, for instance, tree-level unitarity of a massive high-spin particle is achieved in string theory in part because of the three-point vertex, but also because an infinite number of particles of ever-increasing mass and spin propagate as intermediate states. To find which one-graviton vertex is selected by string theory, one must therefore resort to direct calculation.

More specifically, we begin in Sec. II with an investigation of the superstring three-point vertex involving one graviton and two spin- $\frac{7}{2}$ fermions at the first string mass level. Our reasons for this choice are twofold: (i) it is known that difficulties with tree level unitarity do not arise for massive particles of spins less than $\frac{5}{2}$, and (ii) closed string calculations are simplified for states on the leading Regge trajectory-in this case spin- $\frac{7}{2}$ for spacetime fermions at the first mass level. Note that we work in the weak field approximation throughout the paper, and work to linearized order in the gravitational field as we only concentrate on three-point gravitational vertices.

Section III focuses on the "gravitational quadrupole" term, that is the three-point vertex proportional to the Riemann tensor. In Sec. III we compare the results obtained from superstring theory with the predictions of tree-level unitarity. Section IV extends the computation of the threepoint vertex and effective action to states of arbitrary spin, and contains some comments on the implications of our results for tree-level unitarity in strings.

\section{MASSIVE THREE-POINT FUNCTIONS}

We begin by examining the simplest massive string interactions of the type II string, namely, three-point functions at the first mass level. In this case, the (ten-dimensional) closed 
superstring states fall into the $S O(9)$ spin representations ${ }^{1}$ $[44+84+128]_{L} \times[44+84+128]_{R}$. Correspondingly, in four dimensions, such states carry spins up to four. Focusing on spacetime fermions [i.e., the Ramond-Neveu-Schwarz (RNS) sector], we now describe the vertex operator for emission or absorption of states in the $128 \times 44$ of $S O(9)$ and in the $(q, \bar{q})=\left(-\frac{1}{2},-1\right)$ ghost picture. While the string amplitudes are calculated in ten dimensions, the compactification to four dimensions is straightforward on a six-torus, with the 128- and 44-dimensional representations yielding spins up to $\frac{3}{2}$ and 2 , respectively.

Massive vertex operators have been discussed previously in Ref. [3]. Working in the R-NS sector, the vertex operator for the $128 \times 44$ state may be written as

$$
\begin{aligned}
V_{F}^{(-1 / 2,-1)}(z, \bar{z})= & u_{\mu \nu \rho}^{\alpha}(X) S_{\alpha} e^{-\phi / 2} \partial X^{\mu} e^{-\bar{\phi}} \bar{\psi}^{\nu} \bar{\partial} X^{\rho}+v_{\mu \nu \rho}^{\dot{\alpha}}(X) \\
& \times(\gamma \lambda)_{\dot{\alpha}}^{\beta} \psi^{\mu} \psi^{\lambda} S_{\beta} e^{-\phi / 2} e^{-\bar{\phi}} \bar{\psi}^{\nu} \bar{\partial} X^{\rho}
\end{aligned}
$$

In this expression $S_{\alpha}$ and $e^{-\phi / 2}$ represent the spin fields for the two-dimensional fermions $\psi^{\mu}$ and the superconformal ghosts $\beta, \gamma$ respectively. The wave functions $u$ and $v$ are now constrained by demanding that $V_{F}^{(-1 / 2,-1)}(z, \bar{z})$ is Becchi-Rouet-Stora-Tyupin (BRST) invariant, i.e. that $\left[Q, V_{F}^{(-1 / 2,-1)}(z, \bar{z})\right]$ and similarly $\left[\bar{Q}, V_{F}^{(-1 / 2,-1)}(z, \bar{z})\right]$ vanish up to a total derivative. The BRST charge for the type II string theory is given by

$$
\begin{aligned}
Q= & \int d z c(z)\left(-\frac{1}{2} \eta^{\mu \nu} \partial X_{\mu} \partial X_{\nu}-\frac{1}{2} \eta^{\mu \nu} \psi_{\mu} \partial \psi_{\nu}-\frac{3}{2} \beta \partial \gamma-\frac{1}{2} \partial \beta \gamma\right)(z) \\
& +\int d z\left(b c \partial c+\frac{1}{2} \gamma \eta^{\mu \nu} \psi_{\mu} \partial X_{\nu}-\frac{1}{4} b \gamma^{2}\right)(z)
\end{aligned}
$$

and a similar expression for $\bar{Q}$. We find that $\left[Q, V_{F}^{(-1 / 2,-1)}(z, \bar{z})\right]=\partial\left[c(z) V_{F}^{(-1 / 2,-1)}(z, \bar{z})\right]$ and $\left[\bar{Q}, V_{F}^{(-1 / 2,-1)}(z, \bar{z})\right]$ $=\bar{\partial}\left[\bar{c}(\bar{z}) V_{F}^{(-1 / 2,-1)}(z, \bar{z})\right]$ when the wave functions $u$ and $v$ satisfy

$$
\begin{gathered}
\left(\gamma^{\lambda}\right)_{\beta}^{\dot{\beta}} \partial_{\lambda} v_{\mu \nu \rho}^{\dot{\alpha}}(X) \delta_{\dot{\alpha} \dot{\beta}}+\frac{\sqrt{2}}{(D-2)} u_{\mu \nu \rho}^{\alpha}(X) \delta_{\alpha \beta}=0, \quad \partial^{\mu} u_{\mu \nu \rho}^{\alpha}(X)=0, \\
\left(\gamma^{\lambda}\right)_{\alpha \dot{\beta}} \partial_{\lambda} u_{\mu \nu \rho}^{\alpha}(X)+(D-2) v_{\mu \nu \rho}^{\dot{\alpha}}(X) \delta_{\dot{\alpha} \dot{\beta}}=0, \quad\left(\gamma^{\mu}\right)_{\dot{\alpha}}^{\beta} v_{\mu \nu \rho}^{\dot{\alpha}}(X)=0, \\
\left(\gamma^{\mu}\right)_{\alpha \dot{\beta}} u_{\mu \nu \rho}^{\alpha}(X)-(D-2) \partial^{\mu} v_{\mu \nu \rho}^{\dot{\alpha}}(X) \delta_{\dot{\alpha} \dot{\beta}}=0, \quad \partial^{\nu} u_{\mu \nu \rho}^{\alpha}(X)=\partial^{\nu} v_{\mu \nu \rho}^{\dot{\alpha}}(X)=0,
\end{gathered}
$$

where $D=10$ is the space-time dimension. Conformal invariance thus provides equations of motion and gauge conditions for the wave functions $u_{\mu \nu \rho}^{\alpha}(X)$ and $v_{\mu \nu \rho}^{\dot{\alpha}}(X)$. These constraints can be written in a compact form if we combine $u$ and $v$ into a Dirac spinor $\psi=\left(\begin{array}{l}u \\ (D-2) v\end{array}\right)$ :

$$
\begin{gathered}
\gamma^{\lambda} \partial_{\lambda} \psi_{\mu \nu \rho}(X)+\sqrt{2} \psi_{\mu \nu \rho}(X)=0 \\
\gamma^{\mu} \psi_{\mu \nu \rho}(X)=\partial^{\mu} \psi_{\mu \nu \rho}(X)=\partial^{\nu} \psi_{\mu \nu \rho}(X)=\partial^{\rho} \psi_{\mu \nu \rho}(X)=0 .
\end{gathered}
$$

We observe that $\psi_{\mu \nu \rho}(X)$ obeys a massive Dirac equation and a set of gauge conditions. In order to extract the couplings of massive spin- $-\frac{7}{2}$ particles to gravity we need to calculate the three-point scattering amplitude of two spin- $\frac{7}{2}$ fer-

\footnotetext{
${ }^{1}$ Both IIA and IIB theories have identical massive spectra; their interactions, however, are in general distinct.
}

mions and a graviton. To satisfy the superconformal ghost charge condition we consider the graviton vertex operator in the $(q, \bar{q})=(-1,0)$ picture

$$
V_{G}^{(-1,0)}(z, \bar{z})=h_{\mu \nu}(X) \psi^{\mu} e^{-\phi} \bar{\partial} X^{\nu}+\partial_{\lambda} h_{\mu \nu}(X) \psi^{\mu} e^{-\phi} \bar{\psi}^{\lambda} \bar{\psi}^{\nu},
$$

where the graviton wave function obeys $\square h^{\mu \nu}(X)$ $=\partial_{\mu} h^{\mu \nu}(X)=0$. We proceed now to calculate $\left\langle V_{F}^{(-1 / 2,-1)}\left(z_{1}, \bar{z}_{1}\right) V_{G}^{(-1,0)}\left(z_{2}, \bar{z}_{2}\right) V_{F}^{(-1 / 2,-1)}\left(z_{3}, \bar{z}_{3}\right)\right\rangle$.

To calculate superstring three-point functions involving spin fields we use the techniques developed in Ref. [4]. In particular, we note that three-point functions always factorize into a product of a holomorphic and an antiholomorphic piece. We recall that the closed string graviton vertex operator, Eq. (5), is simply a product of separate left- and rightmoving gauge boson vertices. Starting with the holomorphic Ramond sector, we now calculate the three-point function of two massive fermions with a gauge boson. Dynamical issues of massive string states in open string theory were discussed in Ref. [5]. 
The vertex operators which describe massive spin- $\frac{3}{2}$ fermions and massless gauge bosons in the $q=-\frac{1}{2}$ and $q=$ -1 ghost pictures, respectively, are given by

$$
\begin{gathered}
V_{F}^{(-1 / 2)}(z, \bar{z})=u_{\mu}^{\alpha}(X) S_{\alpha} e^{-\phi / 2} \partial X^{\mu}+v_{\mu}^{\dot{\alpha}}(X) \\
\times\left(\gamma_{\lambda}\right)_{\dot{\alpha}}^{\beta} \psi^{\mu} \psi^{\lambda} S_{\beta} e^{-\phi / 2} \\
V_{B}^{(-1)}(z, \bar{z})=A_{\mu}(X) \psi^{\mu} e^{-\phi} .
\end{gathered}
$$

Conformal invariance implies that $A_{\mu}$ and $\psi=\left(\begin{array}{l}u \\ (D-2) v\end{array}\right)$ satisfy

$$
\begin{gathered}
\gamma^{\lambda} \partial_{\lambda} \psi_{\mu}(X)+\sqrt{2} \psi_{\mu}(X)=0, \quad \gamma^{\mu} \psi_{\mu}(X)=\partial^{\mu} \psi_{\mu}(X)=0, \\
\square A^{\mu}(X)=\partial_{\mu} A^{\mu}(X)=0 .
\end{gathered}
$$

The gauge conditions $\gamma \cdot \psi=\partial \cdot \psi=0$ eliminate the spin- $\frac{1}{2}$ components and thus the vector-spinor wave function $\psi_{\mu}^{\alpha}$ describes a pure spin- $\frac{3}{2}$ massive open string state.

Taking into account momentum conservation and the conditions that different polarizations obey, we find

$$
\begin{aligned}
\mathcal{A}_{F F}^{\gamma}\left(\psi_{1 \mu}, k_{1} ; A_{2 \sigma}, k_{2} ; \psi_{3 \mu}, k_{3}\right) \\
=\frac{1}{\sqrt{2}}\left[\bar{\psi}_{1 \mu} \gamma^{\sigma} A_{2 \sigma} \psi_{3}^{\mu}-\bar{\psi}_{1 \mu} \gamma^{\sigma} A_{2 \sigma} \gamma_{+} \psi_{3 \nu} k_{2}^{\mu} k_{2}^{\nu}\right] \\
+i\left[\bar{\psi}_{1 \mu} \gamma_{+} \psi_{3}^{\nu}-\bar{\psi}_{1 \mu} \gamma_{-} \psi_{3}^{\nu}\right] k_{2}^{\mu} A_{2}^{\nu},
\end{aligned}
$$

where $\gamma_{+}\left(\gamma_{-}\right)$projects the Dirac spinor $\psi$ onto its positive (negative) chirality components (in the ten-dimensional sense).

While this is meant to be viewed as the holomorphic component of the closed string amplitude, it also has the interpretation as an open string calculation of a single photon emission from a spin- $\frac{3}{2}$ particle. To this linear order in the gauge field, the corresponding effective field theoretic Lagrangian reproducing the three-point function (8) is given by

$$
\begin{aligned}
\mathcal{L}_{(3 / 2)}= & -\frac{i}{2} \bar{\psi}_{\mu}\left(\gamma^{\lambda} D_{\lambda}+m\right) \psi^{\mu}-\frac{1}{2 m} \bar{\psi}_{\mu} F^{\mu \nu} \psi_{\nu} \\
& +\frac{1}{m^{2}} \bar{\psi}_{\mu} \partial^{\mu} F_{\nu \lambda} \gamma^{\lambda} \gamma_{+} \psi^{\nu}+\cdots,
\end{aligned}
$$

where $m=\sqrt{2}$ and $D_{\mu}=\partial_{\mu}+i A_{\mu}$. The ellipses represent both terms that possibly vanish on-shell and terms which are necessary to implement the physical state constraints (including the introduction of nonphysical degrees of freedom). In the present paper we are mainly interested only in the form of the interaction between massive higher-spin string states and external gravitational or electromagnetic fields and not in the details of the additional terms necessary for the consistency of the effective Lagrangian. ${ }^{2}$

Completing the spin- $\frac{7}{2}$ calculation, we now examine the antiholomorphic sector, corresponding to gauge boson emission from a massive spin-2 boson. The calculation is straightforward, especially for the NS sector in the covariant formalism. The corresponding vertex operators in the ghost picture $q=0$ and $q=-1$ read

$$
V_{B}^{(0)}(z, \bar{z})=A_{\mu}(X) \bar{\partial} X^{\mu}, \quad V_{B}^{(-1)}(z, \bar{z})=\phi_{\mu \nu}(X) e^{-\bar{\phi}} \bar{\psi}^{\mu} \bar{\partial} X^{\nu},
$$

while the resulting amplitude is given by

$$
\begin{aligned}
\mathcal{A}_{B B}^{\gamma}\left(\phi_{1 \mu \nu}, k_{1} ; A_{2 \sigma}, k_{2} ; \phi_{3 \mu \nu}, k_{3}\right) & \\
= & \phi_{1 \mu \nu} \phi_{3 \lambda \sigma}\left[\eta^{\mu \lambda} \eta^{\nu \sigma} A_{2} \cdot k_{3}+4 \eta^{\mu \lambda} k_{2}^{[\nu} A_{2}^{\sigma]}\right. \\
& \left.-\eta^{\mu \lambda} k_{2}^{\nu} k_{2}^{\sigma} A_{2} \cdot k_{3}-2 k_{2}^{\mu} k_{2}^{\lambda} k_{2}^{[\nu} A_{2}^{\sigma]}\right]
\end{aligned}
$$

with corresponding (open string) effective Lagrangian

$$
\begin{aligned}
\mathcal{L}_{(2)}= & -\frac{1}{2} D_{\rho} \phi_{\mu \nu} D^{\rho} \phi^{\mu \nu}-\frac{1}{2} m^{2} \phi_{\mu \nu} \phi^{\mu \nu}+2 i \phi_{\mu \nu} F^{\nu \sigma} \phi^{\mu \sigma} \\
& -\frac{2 i}{m^{2}}\left(\phi_{\mu \nu} \partial^{\nu} F_{\rho \sigma} \partial^{\rho} \phi_{\sigma}^{\mu}-\phi_{\mu \nu} \partial^{\mu} \partial^{\lambda} F_{\nu \sigma} \phi_{\lambda}^{\sigma}\right) .
\end{aligned}
$$

The three-point function for the closed string states $\mathcal{A}_{F F}^{h}$ follows by combining the holomorphic and antiholomorphic three-point functions for the open string states, Eqs. (8) and (11). Note that extracting the spin $-\frac{7}{2}$ amplitude from the tensor product $\mathcal{A}_{F F}^{\gamma(\text { left })} \times \mathcal{A}_{B B}^{\gamma \text { (right) }}$ involves symmetrization on the vector indices arising from the holomorphic and antiholomorphic polarizations. We find

\footnotetext{
${ }^{2}$ See, e.g., Ref. [6] for a discussion about the need to introduce nonphysical degrees of freedom for higher spin string states.
} 


$$
\begin{aligned}
\mathcal{A}_{F F}^{h}\left(\psi_{1 \mu \nu \lambda}, k_{1} ; h_{\mu \nu}, k_{2} ; \psi_{3 \mu \nu \lambda}, k_{3}\right)= & \mathcal{A}_{F F}^{\gamma(\text { left })} \times \mathcal{A}_{B B}^{\gamma(\text { right })} \\
= & \bar{\psi}_{1 \mu \nu \lambda} \gamma^{\sigma} \psi_{3}^{\mu \nu \lambda} h_{\sigma \rho} k_{3}^{\rho}+2 \bar{\psi}_{1 \mu \nu \lambda} \gamma^{\sigma} \psi_{3}^{\mu \nu \rho}\left(h_{\rho \sigma} k_{2}^{\lambda}-h_{\sigma}^{\lambda} k_{2 \rho}\right)+i \sqrt{2}\left(\bar{\psi}_{1 \mu \nu \lambda} \gamma_{+} \psi_{3 \sigma}{ }^{\nu \lambda}\right. \\
& \left.-\bar{\psi}_{1 \sigma \nu \lambda} \gamma_{-} \psi_{3 \mu}{ }^{\nu \lambda}\right) h^{\mu \rho} k_{2}^{\sigma} k_{3 \rho}+2 i \sqrt{2}\left(\bar{\psi}_{1 \mu \nu \lambda} \gamma_{+} \psi_{3 \sigma}{ }^{\nu \rho}-\bar{\psi}_{1 \sigma \nu \lambda} \gamma-\psi_{3 \mu}{ }^{\nu \rho}\right) \\
& \times\left(h^{\mu}{ }_{\rho} k_{2}^{\sigma} k_{2}^{\lambda}-h^{\mu \lambda} k_{2}^{\sigma} k_{2 \rho}\right)-\bar{\psi}_{1 \mu \nu \lambda} \gamma^{\sigma}\left(1+\gamma_{+}\right) \psi_{3 \rho}{ }^{\nu \lambda} h_{\sigma \delta} k_{3}^{\delta} k_{2}^{\mu} k_{2}^{\rho}-\bar{\psi}_{1 \mu \nu \lambda} \gamma^{\sigma}\left(1+2 \gamma_{+}\right) \\
& \times \psi_{3}^{\mu \rho \delta}\left(h_{\sigma \delta} k_{2}^{\nu} k_{2 \rho} k_{2}^{\lambda}-h_{\sigma}{ }^{\lambda} k_{2}^{\nu} k_{2 \rho} k_{2 \delta}\right)-i \overline{2}_{2}\left(\bar{\psi}_{1 \mu \nu \lambda} \gamma_{+} \psi_{3 \sigma}{ }^{\nu \rho}-\bar{\psi}_{1 \sigma \nu \lambda} \gamma_{-} \psi_{3 \mu}{ }^{\nu \rho}\right) \\
& \times h^{\mu \delta} k_{2}^{\sigma} k_{3 \delta} k_{2}^{\lambda} k_{2 \rho}-i \sqrt{2}\left(\bar{\psi}_{1 \mu \nu \lambda} \gamma_{+} \psi_{3 \sigma \rho \delta}-\bar{\psi}_{1 \sigma \nu \lambda} \gamma_{-1} \psi_{3 \mu \rho \delta}\right) \\
& \times\left(h^{\mu \delta} k_{2}^{\sigma} k_{2}^{\nu} k_{2}^{\lambda} k_{2}^{\rho}-h^{\mu \lambda} k_{2}^{\sigma} k_{2}^{\nu} k_{2}^{\delta} k_{2}^{\rho}\right)+\bar{\psi}_{1 \mu \nu \lambda} \gamma^{\sigma} \gamma_{+} \psi_{3 \rho}{ }^{\nu \delta} h_{\sigma \epsilon} k_{3}^{\epsilon} k_{2}^{\mu} k_{2}^{\lambda} k_{2 \delta} k_{2}^{\rho} \\
& +\bar{\psi}_{1 \mu \nu \lambda} \gamma^{\sigma} \gamma_{+} \psi_{3 \rho \delta \epsilon}\left(h_{\sigma}{ }^{\epsilon} k_{2}^{\nu} k_{2}^{\mu} k_{2}^{\lambda} k_{2}^{\delta} k_{2}^{\rho}-h_{\sigma}{ }^{\lambda} k_{2}^{\nu} k_{2}^{\mu} k_{2}^{\epsilon} k_{2}^{\delta} k_{2}^{\rho}\right) .
\end{aligned}
$$

As a result the effective Lagrangian that reproduces this particular three-point function contains terms up to five derivatives. Up to terms that vanish on-shell (but that are nevertheless crucial for the consistency of such a massive higher-spin Lagrangian), we find

$$
\begin{aligned}
\mathcal{L}_{\mathrm{eff}}= & \sqrt{-g}\left[\frac{1}{2} \bar{\psi}_{\mu \nu \lambda}\left(\gamma^{\sigma} \nabla_{\rho}+m\right) \psi^{\mu \nu \lambda}\right. \\
& -\frac{1}{m} \bar{\psi}_{\mu \nu \lambda}\left(R^{\mu \alpha \nu \beta}-\frac{1}{8} R^{\mu \alpha \rho \sigma} \gamma_{\rho \sigma} \eta^{\nu \beta}\right) \psi_{\alpha \beta}{ }^{\lambda} \\
& -\frac{1}{m^{2}}\left(\bar{\psi}_{\mu \nu \lambda} R^{\sigma(\mu \alpha) \rho} \gamma_{\sigma}\left(1+\gamma_{+}\right) \partial_{\rho} \psi_{\alpha}{ }^{\nu \lambda}\right. \\
& \left.-\bar{\psi}_{\mu \nu \lambda} \partial^{(\mu} R^{\alpha) \sigma \nu \beta} \gamma_{\sigma}\left(1+2 \gamma_{+}\right) \psi_{\alpha \beta}{ }^{\lambda}\right) \\
& -\frac{1}{m^{3}}\left(\bar{\psi}_{\mu \nu \lambda} \partial^{(\nu} R^{\beta) \sigma \mu \alpha} \partial_{\alpha} \psi_{\alpha \beta}{ }^{\lambda}\right. \\
& \left.+\bar{\psi}_{\mu \nu \lambda} \partial^{\mu} \partial^{\alpha} R^{\nu \beta \lambda \gamma} \psi_{\alpha \beta \gamma}\right) \\
& -\frac{2}{m^{4}}\left(\bar{\psi}_{\mu \nu \lambda} \partial^{\mu} \partial^{\alpha} R^{\sigma(\beta \nu) \delta} \gamma_{\sigma} \gamma_{+} \partial_{\delta} \psi_{\alpha \beta}{ }^{\lambda}\right. \\
& \left.\left.-\bar{\psi}_{\mu \nu \lambda} \partial^{\nu} \partial^{\delta} \partial^{(\lambda} R^{\gamma) \sigma \mu \rho} \gamma_{\sigma} \gamma_{+} \psi_{\rho \delta \gamma}\right)\right]
\end{aligned}
$$

Several points are in order here. First of all, working with the three-point function, we only obtain information up to linearized order in the graviton $h_{\mu \nu}$. For this reason, at this order there is no distinction between bare and covariant derivatives of the Riemann tensor that appear in Eq. (14). Secondly, this effective Lagrangian is by no means unique, as we are always allowed to shift it by terms vanishing on-shell. In particular, we note that the $\gamma$-transverse condition $\gamma \cdot \psi=0$ allows use of the on-shell identity

$$
\begin{aligned}
\bar{\psi}_{\mu \nu \lambda} R^{\mu \alpha \rho \sigma} \gamma_{\rho \sigma} \eta^{\nu \beta} \psi_{\alpha \beta}{ }^{\lambda}= & 2 \bar{\psi}_{\mu \nu \lambda} R^{\mu \alpha \nu \beta} \psi_{\alpha \beta}{ }^{\lambda} \\
& -\bar{\psi}_{\mu \nu \lambda} R^{\mu \alpha \rho \sigma} \gamma_{\rho \sigma}{ }^{\nu \beta} \psi_{\alpha \beta}{ }^{\lambda},
\end{aligned}
$$

indicating that even the leading two-derivative term is by no means unique. Furthermore, note that the presence of the ten-dimensional chirality projection $\gamma_{+}$for spacetime fermions indicates that, even while massive, such states maintain chiral interactions with gravity. It is at this point where the difference between massive IIA and IIB string states shows up. The complete type II spectrum includes in fact a pair of spin- $\frac{7}{2}$ states at the first mass level, one each from the R-NS and the NS-R sectors. In our conventions, Eq. (14) corresponds to the state from the R-NS sector, while a similar equation with either $\gamma_{-}$or $\gamma_{+}$would describe the state from the NS-R sector for the type IIA or IIB theory, respectively.

Finally, the effective gravitational interactions of string states at higher mass levels have correspondingly higher derivative couplings. Physically, this corresponds to the intuitive notion that highly excited strings are spread out, and hence feel tidal effects arising from the curvature of spacetime. This departure from the minimal coupling prescription leads to violation of the strong equivalence principle [7]. This is just a fact of life; in relativistic quantum field theory particles have an intrinsic size: their Compton wavelength. This makes them behave in some respect as extended objects, sensitive to tidal forces.

\section{NONMINIMAL COUPLING AND THE GRAVITATIONAL QUADRUPOLE}

Nonminimal couplings to the Riemann tensor were discussed in Ref. [2] in the context of point particle field theory, where it was shown that tree-level unitarity for particles of spin $>2$ demands the presence of just such a nonminimal term. The authors of Ref. [2] give a general expression for the required nonminimal addition to the action for both integer and half-integer spins. For the former, the on-shell Lagrangian (also ignoring auxiliary fields) ${ }^{3}$ for a boson of spin $s$ takes the form

\footnotetext{
${ }^{3}$ See, e.g., Ref. [8] for an explicit form of the massive higher spin Lagrangian.
} 


$$
\begin{aligned}
\mathcal{L}= & \frac{1}{2} \phi^{(s)}\left(\nabla_{\mu} \nabla^{\mu}-m^{2}\right) \phi^{(s)}+\frac{s(s-1)}{2} \phi_{\mu \nu}^{(s)} . R^{\mu \alpha \nu \beta} \phi_{\alpha \beta}^{(s)} \ldots \\
& +\cdots
\end{aligned}
$$

The resulting equation of motion for $\phi^{(s)}$ may then be expressed as

$$
\left(\nabla_{\mu} \nabla^{\mu}-m^{2}\right) \phi^{(s)}+\left[R_{\mu \nu \lambda \sigma} \frac{1}{2} \Sigma^{\mu \nu} \frac{1}{2} \Sigma^{\lambda \sigma}\right] \phi^{(s)}+\cdots=0
$$

where ellipses indicate terms vanishing on shell, and $\Sigma^{\mu \nu}$ are the Lorentz generators in the spin- $s$ representation,

$$
\left(\Sigma^{\mu \nu}\right)_{\{\alpha\}}\{\beta\}=2 s \delta_{\left[\alpha_{1}\right.}^{[\mu} \eta^{\nu]\left[\beta_{1}\right.} \delta_{\left.\alpha_{2} \cdots \alpha_{s}\right]}^{\left.\beta_{2} \cdots \beta_{s}\right]},
$$

where all symbols are antisymmetric with weight 1 .

On the other hand, for spin- $\left(n+\frac{1}{2}\right)$ fermions in four dimensions, the nonminimal Lagrangian is

$$
\begin{aligned}
\mathcal{L}= & \bar{\psi}^{(n)}\left(\gamma^{\mu} \nabla_{\mu}-m\right) \psi^{(n)}+\frac{n(n-1)}{2 m} \bar{\psi}_{\mu \nu}^{(n)} \ldots R^{+\mu \alpha \nu \beta} \psi_{\alpha \beta}^{(n) \cdots} \\
& +\cdots
\end{aligned}
$$

(again only up to terms vanishing on-shell). Here $R_{\mu \nu \lambda \sigma}^{+}$ $=R_{\mu \nu \lambda \sigma}+\frac{1}{2} R_{\mu \nu \alpha \beta} \gamma_{\lambda \sigma}^{\alpha \beta}$ is a feature of the four-dimensional theory. Taking the first order equation of motion from Eq. (19) and multiplying by $\left(\gamma^{\mu} \nabla_{\mu}+m\right)$, we obtain the second order equation

$$
\left(\nabla_{\mu} \nabla^{\mu}-m^{2}\right) \psi^{(n)}+\left[R_{\mu \nu \lambda \sigma} \frac{1}{2} \Sigma^{\mu \nu} \frac{1}{2} \Sigma^{\lambda \sigma}\right] \psi^{(n)}+\cdots=0
$$

where this time

$$
\left(\Sigma^{\mu \nu}\right)_{\{\alpha\}}^{\{\beta\}}=\frac{1}{2} \gamma^{\mu \nu} \delta_{\left[\alpha_{1} \cdots \alpha_{n}\right]}^{\left[\beta_{1} \cdots \beta_{n}\right]}+2 n \delta_{\left[\alpha_{1}\right.}^{[\mu} \eta^{\nu]\left[\beta_{1}\right.} \delta_{\left.\alpha_{2} \cdots \alpha_{n}\right]}^{\left.\beta_{2} \cdots \beta_{n}\right]}
$$

is the Lorentz generator in the spin- $\left(n+\frac{1}{2}\right)$ representation.

We now see from Eqs. (17) and (20) that both integer and half-integer spin fields have identical forms for the preferred nonminimal coupling resulting from tree-level unitarity concerns. Furthermore, since the Riemann coupling has the form of a gravitational quadrupole moment, these results of Ref. [2] are suggestive of a gravitational version of the corresponding statement of " $g=2$ "' as a natural value for the gyromagnetic ratio for electromagnetic couplings [9].

The above discussion suggests the definition of a gravitational quadrupole " $h$ factor" that may be determined from the equations of motion according to

$$
\left(\nabla_{\mu} \nabla^{\mu}-m^{2}\right) \varphi+h\left[R_{\mu \nu \lambda \sigma} \frac{1}{2} \Sigma^{\mu \nu} \frac{1}{2} \Sigma^{\lambda \sigma}\right] \varphi+\cdots=0 .
$$

While the $h$ factor may equally well be defined in terms of the nonminimal coupling of $\varphi$ to the Riemann tensor in the
Lagrangian, such a definition is complicated by the fact that there is an inherent ambiguity in the minimal coupling prescription itself (which is not present for the equations of motion). Using this definition, the results of Ref. [2] may be concisely summarized by the statement that $h=1$ is the preferred value of the $h$ factor based on the above field theory considerations.

Turning to the spin- $\frac{7}{2}$ Lagrangian (14) it is clear that it cannot be written in the "preferred" form of Eq. (19), even through the use of the on-shell manipulation (15). ${ }^{4}$ As a result this provides evidence that $h \neq 1$ for massive string states in general. In order to determine the spin- $\frac{7}{2} h$ factor, we first make use of Eq. (15) and work in four dimensions to note that the second order equation of motion arising from Eq. (14) has the form

$$
\begin{aligned}
& \left(\nabla_{\rho} \nabla^{\rho}-m^{2}\right) \psi^{\mu \nu \lambda}+3\left(R^{\mu \alpha \nu \beta}+\frac{1}{2} R^{\mu \alpha \rho \sigma} \gamma_{\rho \sigma} \eta^{\nu \beta}\right) \psi_{\alpha \beta}{ }^{\lambda} \\
& +\cdots=0 .
\end{aligned}
$$

On the other hand, for spin $\frac{7}{2}$, Eq. (22) gives instead

$$
\begin{aligned}
& \left(\nabla_{\rho} \nabla^{\rho}-m^{2}\right) \psi^{\mu \nu \lambda}+6 h\left(R^{\mu \alpha \nu \beta}+\frac{1}{4} R^{\mu \alpha \rho \sigma} \gamma_{\rho \sigma} \eta^{\nu \beta}\right) \psi_{\alpha \beta}{ }^{\lambda} \\
& \quad+\cdots=0
\end{aligned}
$$

which clearly has a different Lorentz structure. What this indicates is that, even when restricted to on-shell interactions, there are in fact two possible distinct Lorentz-invariant and parity conserving interactions that may be written in terms of the Riemann coupling. Thus a single " $h$ factor" is insufficient, and in fact two parameters are necessary to fully characterize this lowest order nonminimal interaction.

On the other hand, working in the Newtonian limit, we find that both $R^{\mu \alpha \nu \beta} \psi_{\alpha \beta} \ldots$ and $\left(R^{\mu \alpha \rho \sigma} \gamma_{\rho \sigma} \eta^{\nu \beta}\right) \psi_{\alpha \beta} \ldots$ reduce to the same form, related to the (nonrelativistic) quadrupole moment $Q^{i j}$. In particular, in four dimensions, the components of the Riemann tensor are given in terms of the Newtonian potential $\phi$ as

$$
\begin{gathered}
R_{0 i 0 j}=\partial_{i} \partial_{j} \phi \\
R_{i j k l}=\delta_{i k} \partial_{j} \partial_{l} \phi+\delta_{j l} \partial_{i} \partial_{k} \phi-\delta_{i l} \partial_{j} \partial_{k} \phi-\delta_{j k} \partial_{i} \partial_{l} \phi .
\end{gathered}
$$

Furthermore, nonrelativistically, the transverse and $\gamma$-transverse conditions on $\psi_{\mu \nu} \ldots$ give both $\psi_{0} \ldots$ $=O(p / M) \psi_{i} \ldots \ll 1$ and $\gamma^{i} \psi_{i} \ldots \ll 1$. Thus we find

$$
R^{\mu \alpha \nu \beta} \psi_{\alpha \beta \cdots} \rightarrow R_{i k j l} \psi^{k l \cdots}=-2 \partial_{(i} \partial^{k} \phi \psi_{j)}{ }^{k \cdots}+\delta_{i j} \partial_{k} \partial_{l} \phi \psi^{k l \cdots}
$$

where the last term, having a trace form, does not contribute diagonally to the leading spin- $\frac{7}{2}$ quadrupole interaction, but instead gives an off-diagonal interaction between spins $\frac{7}{2}$ and $\frac{3}{2}$. For the other possibility, we find instead

\footnotetext{
${ }^{4}$ The factor of $\frac{1}{2}$ arises in Eq. (14) because there $\psi_{\mu \nu \lambda}$ is a Majorana spinor, while in Eq. (19) $\psi^{(n)}$ is a Dirac spinor.
} 


$$
\begin{aligned}
& \left(R^{\mu \alpha \rho \sigma} \gamma_{\rho \sigma} \eta^{\nu \beta}\right) \psi_{\alpha \beta \cdots} \\
& \quad \rightarrow R_{i k l m} \gamma^{l m} \psi^{k^{\cdots}}=-4 \partial_{i} \partial_{k} \phi \psi^{k^{\cdots}}+2 \gamma^{i}\left(\partial_{k} \partial_{l} \phi \gamma^{k} \psi_{l \cdots}\right) .
\end{aligned}
$$

This time the last term (having a $\gamma$-trace form) corresponds to an off-diagonal interaction between spins $\frac{7}{2}$ and $\frac{5}{2}$.

With the above in mind, in practice we define the " $h$ factor" of Eq. (22) only in the Newtonian limit (in other words focusing on angular momentum and not Lorentz generators). As a result, the diagonal spin- $\frac{7}{2}$ equation of motion (23) reduces to

$$
\left(\nabla_{\rho} \nabla^{\rho}-m^{2}\right) \psi^{i j k}-6 \partial^{(i} \partial_{l} \phi \psi_{l}^{j k)}+\cdots=0,
$$

where ellipses now also include off-diagonal interactions with lower-spin states (that are always present and fall on the subleading Regge trajectories). Contrasting this with

$$
\left(\nabla_{\rho} \nabla^{\rho}-m^{2}\right) \psi^{i j k}-9 h \partial^{(i} \partial_{l} \phi \psi_{l}^{j k)}+\cdots=0,
$$

which follows from Eq. (24), finally allows us to determine that $h=\frac{2}{3}$ for this particular massive spin- $\frac{7}{2}$ string state.

At this point we must clarify an apparent paradox. The nonrelativistic formula for the quadrupole moment is, in space-time dimension $D$ :

$$
Q^{i j}=\int d^{D-1} x\left[(D-1) x^{i} x^{j}-\delta^{i j} x^{2}\right] T^{00}, \quad i, j=1, \ldots, D-1 .
$$

The action of particles of spin $s>\frac{1}{2}$ can have a nonminimal coupling proportional to the Einstein tensor:

$$
\Delta S=\int d^{D} x B^{\mu \nu} G_{\mu \nu},
$$

where $B^{\mu \nu}$ is a bilinear in the spin-s field. To linear order in the gravitational field, this induces the following change in the stress energy tensor:

$$
T^{00}=\partial_{i} \partial_{j} B^{i j}+\cdots, \quad T_{i}^{i}=(3-D) \partial_{i} \partial_{j} B^{i j}+\cdots .
$$

Here ellipses stands for terms that do not contribute to the gravitational quadrupole. Substituting this equation into formula (30) we obtain a nonzero change of the quadrupole, induced by terms that vanish on the Einstein shell. ${ }^{5}$ The solution to this paradox is that Eq. (30) is a good definition of the quadrupole only for nonrelativistic matter. In more general cases this definition is wrong, even in the Newtonian limit. The correct definition is obtained by computing the energy of the particle in a static, slowly varying external Newtonian potential $\phi$. The change in energy due to the quadrupole is

$$
\Delta H=\frac{1}{2(D-1)} Q^{i j} \partial_{i} \partial_{j} \phi .
$$

This formula is insensitive by construction to all terms that vanish on the Einstein shell. In the Newtonian limit, the metric is $g_{00}=-1-2 \phi, g_{i j}=\{1-[2 /(D-3)] \phi\} \delta_{i j}$, all other terms vanish. This induces the following change in the energy:

$$
\Delta H=\int d^{D-1} x \phi\left(T^{00}+\frac{1}{D-3} T^{i i}\right) .
$$

Thus, the correct expression for the quadrupole is obtained by replacing $T^{00}$ with $T^{00}+(D-3)^{-1} T_{i}^{i}$ in Eq. (30). By substituting Eq. (32) into this new expression we find that the contribution to the quadrupole due to nonminimal coupling to the Ricci tensor vanishes, as it should. Equation (30) can be used in the Newtonian limit only when $T_{i}^{i} \approx 0$, as it holds, for instance, in macroscopic nonrelativistic matter.

\section{GENERALIZATION TO HIGHER SPIN}

The results for the massive spin- $\frac{7}{2}$ state are easily generalized to arbitrary massive higher spin string states interacting with a graviton. As usual, the three-point function factorizes into separate holomorphic and antiholomorphic parts. For simplicity we focus on states on the leading Regge trajectory, namely states of spin $n+\frac{1}{2}$ and $n+1$, respectively, for the R and NS sectors at mass level $n$.

In the Ramond sector, the spin- $\left(n+\frac{1}{2}\right)$ vertex operator is given by

$$
\begin{aligned}
V_{F}^{(-1 / 2)}(z, \bar{z}) & \\
= & u_{\mu_{1} \cdots \mu_{n}}^{\alpha}(X) S_{\alpha} e^{-\phi / 2} \partial X^{\mu_{1} \cdots \partial X^{\mu_{n}}} \\
& \quad+v_{\mu_{1} \cdots \mu_{n}}^{\dot{\alpha}}(X)\left(\gamma_{\lambda}\right)_{\dot{\alpha}}^{\beta} \psi^{\mu_{1}} \psi^{\lambda} S_{\beta} e^{-\phi / 2} \partial X^{\mu_{2} \cdots \partial X^{\mu_{n}}}
\end{aligned}
$$

The resulting three-point function $\mathcal{A}_{F F}^{\gamma}$ contains terms up to $O\left[\left(\alpha^{\prime} k^{2}\right)^{n}\right]$. To lowest order, we find

\footnotetext{
${ }^{5}$ Terms proportional to the scalar curvature tensor do not contribute to the quadrupole.
} 


$$
\begin{aligned}
& \mathcal{A}_{F F}^{\gamma}\left(\psi_{1}, k_{1} ; A_{2 \sigma}, k_{2} ; \psi_{3}, k_{3}\right)=\frac{n !}{\sqrt{2}}\left[\bar{\psi}_{1_{\mu_{1}} \cdots \mu_{n}} A_{2 \sigma} \gamma^{\sigma} \psi_{3}^{\mu_{1} \cdots \mu_{n}}-i \frac{2 n}{m}\left(\bar{\psi}_{1 \alpha \mu_{2} \cdots \mu_{n}} \gamma_{-} \psi_{3 \beta}{ }^{\mu_{2} \cdots \mu_{n}}\right.\right. \\
& \left.\left.-\bar{\psi}_{1 \beta \mu_{2} \cdots \mu_{n}} \gamma_{+} \psi_{3 \alpha}{ }^{\mu_{2} \cdots \mu_{n}}\right) k_{2}^{\alpha} k_{2}^{\beta}+\cdots\right] \text {, }
\end{aligned}
$$

where $m=\sqrt{2 n}$ is the mass of the $n$th excited level.

Prior to examining the closed string graviton amplitude, we note that the effective field theoretic Lagrangian which reproduces this three-point function for the open string may be written as

$$
\begin{aligned}
\mathcal{L}_{n+1 / 2}^{\text {(open) }}= & -\frac{i}{2} \bar{\psi}_{\mu_{1} \cdots \mu_{n}}\left(\gamma^{\sigma} D_{\sigma}+m\right) \psi^{\mu_{1} \cdots \mu_{n}} \\
& -\frac{1}{2 m} \sum_{i=1}^{n} \bar{\psi}_{\mu_{1} \cdots \mu_{n}} F^{\mu_{i} \nu_{i}} \psi^{\mu_{1} \cdots{ }_{\nu_{i}} \cdots \mu_{n}+\cdots}
\end{aligned}
$$

From the form of the Lagrangian we can verify that the the gyromagnetic ratio $g$ for all such massive fermionic open string states on the leading Regge trajectory is equal to 2 [9].

For the spin- $(n+1)$ state in the NS sector, we find, on the other hand, the leading behavior for the three-point function

$$
\begin{aligned}
\mathcal{A}_{B B}^{\gamma}\left(\xi_{1}, k_{1} ; \zeta_{2}, k_{2} ; \xi_{3}, k_{3}\right) & \\
= & n ! \xi_{1 \alpha \mu_{1} \mu_{2} \cdots \mu_{n}} \xi_{3 \beta \nu_{1}}{ }^{\mu_{2} \cdots \mu_{n}} \zeta_{2 \lambda}\left[\left(\eta^{\alpha \beta} k_{3}^{\lambda}-\eta^{\alpha \lambda} k_{2}^{\beta}\right.\right. \\
& \left.\left.+\eta^{\beta \lambda} k_{2}^{\alpha}\right) \eta^{\mu_{1} \nu_{1}}+n\left(\eta^{\nu_{1} \lambda} k_{2}^{\mu_{1}}-\eta^{\mu_{1} \lambda} k_{2}^{\nu_{1}}\right) \eta^{\alpha \beta}+\cdots\right],
\end{aligned}
$$

where once again $m=\sqrt{2 n}$. In general, as for the fermions, the complete expression contains terms up to $O\left[\left(\alpha^{\prime} k^{2}\right)^{n}\right]$. Viewed as an open string amplitude, the corresponding effective Lagrangian has the form

$$
\begin{aligned}
\mathcal{L}_{n+1}^{(\text {open })}= & -\frac{1}{2} D_{\lambda} \phi_{\mu_{0} \cdots \mu_{n}} D^{\lambda} \phi^{\mu_{0} \cdots \mu_{n}}-\frac{1}{2} m^{2} \phi_{\mu_{0} \cdots \mu_{n}} \phi^{\mu_{0} \cdots \mu_{n}} \\
& +i \sum_{i=0}^{n} \phi_{\mu_{0} \cdots \mu_{n}} F^{\mu_{i} \nu_{i}} \phi^{\mu_{0} \cdots}{ }_{\nu_{i}}^{\mu_{i} \cdots \mu_{n}}+\cdots
\end{aligned}
$$

As expected, this indicates that the nonminimal electromagnetic coupling to $F_{\mu \nu}$ gives precisely $g=2$ for the open string.

Turning now to the closed string, combining Eqs. (36) and (38) and symmetrizing on the vector indices (corresponding to the maximal spin state at mass level $n$ ) gives the following on-shell form of the effective Lagrangian:

$$
\begin{aligned}
\mathcal{L}_{2 n+3 / 2}= & \sqrt{-g}\left[\frac{1}{2} \bar{\psi}^{(2 n+1)}\left(\gamma^{\mu} \nabla_{\mu}+m\right) \psi^{(2 n+1)}\right. \\
& -\frac{n(n+1)}{2 m} \bar{\psi}_{\mu_{0} \mu_{1} \cdots \mu_{2 n}}\left(R^{\mu_{0} \nu_{0} \mu_{1} \nu_{1}-\frac{1}{4(n+1)}}\right. \\
& \left.\times R^{\mu_{0} \nu_{0} \lambda \sigma} \gamma_{\lambda \sigma} \eta^{\mu_{1} \nu_{1}}\right) \psi_{\nu_{0} \nu_{1}}^{\left.\mu_{2} \cdots \mu_{2 n}+\cdots\right]}
\end{aligned}
$$

The resulting second order equation for $\psi$ takes the form

$$
\begin{gathered}
\left(\nabla_{\rho} \nabla^{\rho}-m^{2}\right) \psi_{\mu_{0} \mu_{1} \ldots}+\left[2\left(S_{L}-\frac{1}{2}\right)\left(S_{R}-\frac{1}{2}\right) R^{\mu_{0} \nu_{0} \mu_{1} \nu_{1}}\right. \\
\left.+\frac{1}{2}\left(S-\frac{1}{2}\right) R^{\mu_{0} \nu_{0} \lambda \sigma} \gamma_{\lambda \sigma} \eta^{\mu_{1} \nu_{1}}\right] \psi_{\nu_{0} \nu_{1} \ldots}+\cdots=0
\end{gathered}
$$

where $S_{L}=n+1$ and $S_{R}=n+\frac{1}{2}$ are the components of the spin contributed by left and right movers on the world sheet, respectively, and $S=S_{L}+S_{R}\left(=2 n+\frac{3}{2}\right)$ is the total (spacetime) spin. Using this suggestive form of the nonminimal interaction, we find the corresponding $h$ factor to be

$$
h=\frac{2 S_{L} S_{R}}{\left(S-\frac{1}{2}\right)^{2}} \text { (half-integer spins). }
$$

A similar calculation in the NS-NS sector (also on the leading Regge trajectory) gives similarly

$$
h=\frac{2 S_{L} S_{R}}{S(S-1)} \text { (integer spins). }
$$

Note the resemblance to the string $g$-factor result [10]

$$
g_{L}=2 \frac{\left\langle S_{z}^{R}\right\rangle}{S_{z}}, \quad g_{R}=2 \frac{\left\langle S_{z}^{L}\right\rangle}{S_{z}} .
$$

Based on the factorization of the graviton three-point function in terms of holomorphic and antiholomorphic gauge boson amplitudes and the $g=1$ result for all massive open string states, it is now apparent that the $h$-factor result, Eqs. (42) and (43), is equally valid for all massive string states and is not restricted to those on the leading Regge trajectory.

\section{CONCLUSIONS}

In this paper we have examined the three-point couplings of massive higher-spin string states with gravity. Focusing 
on the spin- $\frac{7}{2}$ state at the first mass level of the type II string, we have obtained an effective Lagrangian reproducing all on-shell interactions to linearized order in the graviton. In particular, this effective Lagrangian contains a nonminimal two-derivative coupling of the form $(\bar{\psi} R \psi)$, which was examined by the authors of Ref. [2] in the context of tree-level unitarity. Since this has the form of a gravitational quadrupole interaction, we have defined the gravitational " $h$ factor' (in analogy with the electromagnetic $g$ factor) and demonstrated that Ref. [2] gives $h=1$ as a preferred value of the $h$ factor in field theory.

On the other hand, in a string theory, $h$ is determined from a combination of left- and right-moving components of the spin for massive closed string states. Although generically $h \neq 1$ in string theory in contrast to the field theoretic result, this is certainly not a disaster for string theory. In field theory the tree-level unitarity results hold for a single massive higher spin particle interacting with gravity, while in string theory tree-level unitarity is achieved not only by the threepoint interaction but also because a whole tower of states of arbitrarily large masses and spins propagate as intermediate states.

\section{ACKNOWLEDGMENTS}

I.G. would like to thank S. Samuel for useful discussions. M.P. would like to thank S. Deser for illuminating notes on the correct definition of the gravitational quadrupole, that lay too long in a drawer. This work was supported in part by the Department of Energy under Contract No. DE-FG0291ER40651-TASK B, and by NSF under Grant No. PHY9722083.
[1] C. Aragone and S. Deser, Phys. Lett. 86B, 161 (1979); M. Duff and S. M. Christensen, Nucl. Phys. B154, 301 (1979).

[2] M. Porrati, Phys. Lett. B 304, 77 (1993); A. Cucchieri, M. Porrati, and S. Deser, Phys. Rev. D 51, 4543 (1995).

[3] I. G. Koh, W. Troost, and A. Van Proeyen, Nucl. Phys. B292, 201 (1987); Y. Tanii and Y. Watabiki, ibid. B316, 171 (1989); S. Weinberg, Phys. Lett. 156B, 309 (1985).

[4] V. A. Kostelecky, O. Lechtenfeld, S. Samuel, and S. Watamura, Nucl. Phys. B288, 173 (1987); S. Samuel, ibid. B294, 365 (1987).

[5] J. M. F. Labastida and M. Vozmediano, Nucl. Phys. B312, 308 (1989); P. C. Argyres and C. R. Nappi, Phys. Lett. B 224, 89 (1989); I. L. Buchbinder, V. A. Krykhtin, and V. D. Pershin, ibid. 348, 63 (1995).

[6] M. Evans and I. Giannakis, Phys. Rev. D 44, 2467 (1991).
[7] F. A. Berends and R. Gastmans, Ann. Phys. (N.Y.) 98, 225 (1976); K. A. Milton, Phys. Rev. D 15, 2149 (1977); I. T. Drummonds and S. J. Hathrell, ibid. 22, 343 (1980); R. D. Daniels and G. M. Shore, Nucl. Phys. B425, 634 (1994); P. F. Mende, in String Quantum Gravity and Physics at the Planck Scale, Proceedings of the Erice Workshop, Erice, Italy, 1992, edited by N. Sanchez (World Scientific, Singapore, 1993); R. Lafrance and R. C. Myers, Phys. Rev. D 51, 2584 (1995).

[8] L. P. S. Singh and C. R. Hagen, Phys. Rev. D 9, 898 (1974); 9, 910 (1974).

[9] S. Ferrara, M. Porrati, and V. Telegdi, Phys. Rev. D 46, 3529 (1992).

[10] J. G. Russo and L. Susskind, Nucl. Phys. B437, 611 (1995); A. Sen, ibid. B440, 421 (1995). 